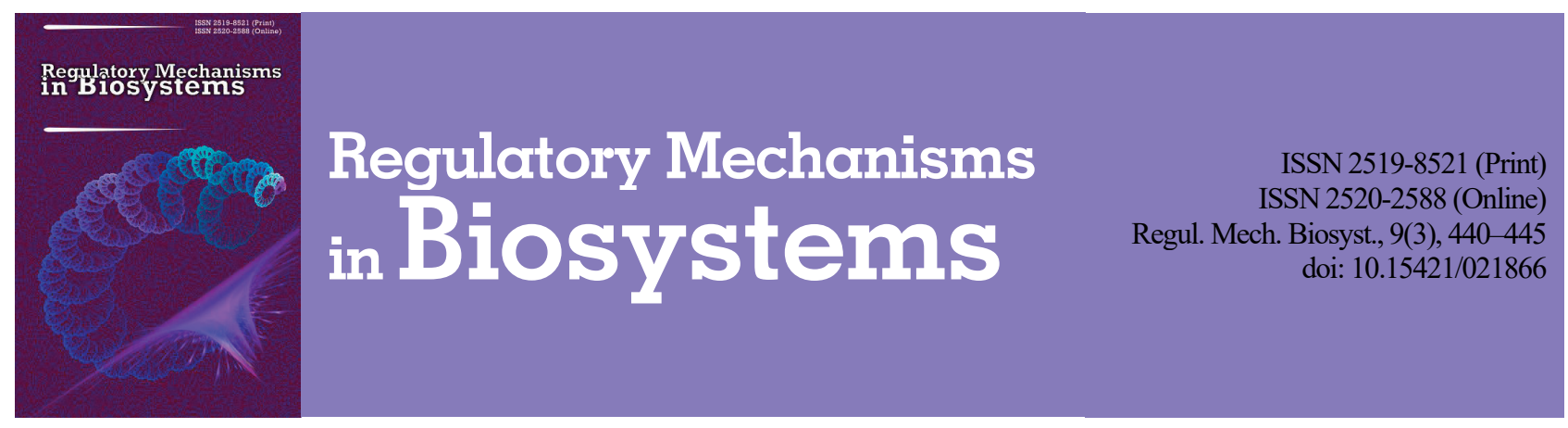

\title{
Influence of solution of lactoprotein with sorbitol on ultrastructural changes in lungs of rats in the condition of burn shock
}

\author{
A. O. Ocheretnyuk, O. V. Palamarchuk, D. A. Lysenko, G. I. Vashchuk, G. I. Stepanyuk \\ National Pirogov Memorial Medical University, Vinnytsya, Ukraine
}

Article info

Received 24.07.2018

Received in revised form

21.08.2018

Accepted 23.08.2018

National Pirogov Memorial

Medical University,

Pirogov st., 56 ,

Vinnytsya, 21000, Ukraine.

Tel.: +38-067-90-90-930.

E-mail:

anna.ocheretniuk@gmail.com

\begin{abstract}
Ocheretnyuk, A. O., Palamarchuk, O. V., Lysenko, D. A., Vashchuk, G. I., \& Stepanyuk, G. I. (2018). Influence of solution of lactoprotein with sorbitol on ultrastructural changes in lungs of rats in the condition of burn shock. Regulatory Mechanisms in Biosystems, 9(3), 440-445. doi:10.15421/021866
\end{abstract}

This article gives a theoretical substantiation and a new experimental solution of a scientific problem aimed at increasing the effectiveness of pharmacotherapy on the morphofunctional state of the lungs of rats under conditions of burn shock by using a combined colloid-hyperosmolar infusion solution - lacto-protein with sorbitol. The administration of the test solution at a dose of $10 \mathrm{ml} / \mathrm{kg}$ for 7 days in rats with modelled burn shock reduced ultrastructural changes in the lungs triggered by burn shock. It has been proved that in the conditions of shock, colloid-hyperosmolar infusion lacto-protein with sorbitol solution facilitates the restoration of vascular endothelium and fluid retention in the microcirculatory channel and improves the morphofunctional state of the aerohematic barrier of the lungs, stimulates the activity of the alveolar macrophages and the secretory function of the type II alveolocytes producing surfactant. At day 7 of burn shock, when $0.9 \%$ of $\mathrm{NaCl}$ was injected, significant changes were observed in the respiratory unit: part of the alveoli had considerably enhanced clearance of blood capillaries, which had platelets, neutrophils and altered forms of erythrocytes. At day 7 of burn shock in the lungs of the rats given an infusion of colloid-hyperosmolar solution lactoprotein with sorbitol, the ultrastructure of the components of the lung cells had improved in comparison with 3 days. Luminosity of the hemocapillary parts was moderate, mainly with erythrocytes. The walls of endothelial cells had elongated nuclei with invaginations of nuclear membranes and clear contours. Their cytoplasmic regions were not widespread, with moderate electron densities. In type II alveolocytes, during this experiment, a lower degree of damage to the nucleus and organelles in the cytoplasm was established, and there were signs of a renewal of the secretory function of these cells. In the cytoplasm, hypertrophied mitochondria with clear crystals, different sizes of secretory granules, which had a different density, indicating their formation, were observed. According to the magnitude of the cytoprotective effect on lung cells under conditions of burn shock, the lactoprotein with sorbitol solution was shown to be superior in comparison with the physical solution $(0.9 \% \mathrm{NaCl})$. The study of functional, biochemical and molecular genetic parameters that characterize the state of the aerohematic barrier under the conditions of using lactoprotein with sorbitol solution in the case of burn injuries of the skin will allow researchers to comprehensively evaluate the mechanisms of the pulmonary protective effect of this preparation and to experimentally substantiate the expediency of its use in clinical practice for pharmaco-correction of burn shock.

Keywords: thermal damage; skin; infusion therapy; pulmonary injuries.

\section{Introduction}

Burn injuries and the various types of damage to the organs and systems of the body to which it leads is one of the pressing problems of modern medicine throughout the world and in Ukraine, in particular (Fuzaylov et al., 2015; Gamelli et al., 2015). Burn illness is a symptom complex when there are functional and morphological changes in vital organs and systems, violation of metabolic and neurohumoral processses, burn shock (BS) (Snell et al., 2013; Nielson et al., 2017). The development of pulmonary complications contributes to an increase in mortality of up to $50 \%$, which raises the issue of early diagnosis and prevention of lung injuries in burn shock as an important problem of combustiology (Cox et al., 2015).

The lungs are a target organ in burn chock, damage to lungs occurs as a result of the development of several pathogenesis units, which constitute a mosaic picture of severe damage and violation of the whole homeostasis of the body and require effective pharmacotherapy, in the first place, adequate correction of disorders in the early stages of burn injury (Porter et al., 2016). Pathogenesis of development and cellular mechanisms of lung damage is the least studied aspect of this problem, which, in turn, hinders the development of adequate and sufficiently effective methods of treatment of this pathology (Kaddoura et al., 2017). In the literature, morphofunctional changes in the structure of the lungs are not sufficiently studied, attention is not paid to the dynamics of these disorders, depending on the period of burn disease, and their relationship, which requires further research (Jacob et al., 2015; Sousse et al., 2015).

An analysis of the clinical picture of lung injury on the background of burns indicates the diversity of its forms, but more often two of its main varieties are distinguished (Cox et al., 2015). The first of these is associated with the effect of high temperatures on the mucous membrane of the respiratory tract. This form of lung inflammation according to some authors stands out as "primary burn pneumonia" (Shaver \& Bastarache, 2014). One of the targets of these factors is the lung cells in which there is a decrease in the activity of synthetic processes, a more active response to apoptotic stimuli, their functioning is disturbed, which creates the preconditions for the development of clinical manifestations of lung injury on the background of thermal damage (Herold et al., 2015).

The development of lung injury is also associated with the polyfactor effect of thermal damage and has the character of a chain reaction. Burn shock causes the destruction of the phospholipid layer of mem- 
branes under the action of histamine and kinins and violations of tissue respiration, permeability of the alveolar-capillary membranes, edema and fluid exudation from the vascular bed to the interstitial space and lumen of the alveoli, lung tissues, vascular spasm, small thrombi and plasmatic percolation, neutrophil infiltration, diffuse damage to the walls of the alveoli, hyaline dystrophy (Jacob et al., 2015). Oxygen-metabolic explosion of leukocytes becomes pathological, damaging cells and macromolecular compounds. In the pathogenesis of burn shock, the impairment of the hemodynamics of the small circle of blood circulation and the gas transportation function of the lungs is important. The pronounced disturbances of peripheral blood flow in certain areas of the lungs contribute to the formation of infiltrates, and later bronchopneumonia. In a morphological study of lungs (Sousse et al., 2015), it is possible to observe in the alveolus a protein-rich fluid with acuminate alveolocytes, macrophages, red blood cells, and fibrin cones. In cases of burn shock, functional activity decreases and the alveolar macrophage ultrastructure changes (Zhang et al., 2018). On the basis of violations of hemodynamics and rheological properties of blood, as well as hypoxia, action of biogenic amines and bacterial toxins in the lungs, dystrophic changes take place in the level of necrosis (Shaver \& Bastarache, 2014).

The above changes have a certain sequence and at a certain stage of burn shock develop an irreversible nature, which requires timely correction or preventive intervention. It is important to use means that reduce the toxic effects of burn injury, or completely alleviate the negative factors, this being the goal of modern pathogenetic therapy in burn injury (Abdullahi \& Jeschke, 2014; Snell et al., 2013; Kaddoura et al., 2017). The pathogenesis of development and cellular mechanisms of damage are the least studied, especially in the period after the day of injury, which, in turn, hinders the development of adequate and sufficiently effective methods of treatment of this pathology.

The high incidence of lung injury as a result of burn injury, the severity of this pathology, the high percentage of lung injury in this case, in particular the development of acute respiratory distress syndrome, and the known property of infusion solutions based on hyperosmolytic colloidal solutions, along with the correction of hypovolemic shock and septicemia, to suppress the increase in the amount of fluid in the lungs, without disturbing the gas exchange in them, gives relevance to the research and study of new combined colloid-hyperosmolytic infuseon solutions (Abdullahi \& Jeschke, 2014).

One such drug is a solution of lactoprotein with sorbitol (LPS), which was created at the Lviv Institute of Blood Pathology and Transfusion Medicine (Covalchuk \& Cherkasov, 2014). The results of preclinical study of the drug established its ability to support the vital functions of the body of rats and provide organoprotective effects in the conditions of experimental burn injuries of the skin (Cherkasov et al., 2015). The effect of the drug on ultrastructural changes in the lungs in the period of burn shock is unknown, which necessitates research in this direction.

The goal of our research was to find out the effect of the solution of lactoprotein with sorbitol on the ultrastructural organization of pulmonary tissues in rats with burn shock (BS).

\section{Materials and methods}

Experimental studies were performed on 256 white male rats weighing 160-180 $\mathrm{g}$ in accordance with the requirements of modern standards, as reflected in the international rules of the "European Convention for the Protection of Vertebrate Animals", the methodological recommendations of the Ministry of Health of Ukraine on "Preclinical Research of Medicinal Products" (Directive 2010/63/EC of the European Parliament and of the Council of 22 September 2010 on the Protection of Animals used for Scientific Purposes, 2010).

The animals were catheterized and divided into three groups: I group - intact animals, II, III groups - rats with BS, which were infused respectively: $0.9 \% \mathrm{NaCl}$ solution, lactoprotein with sorbitol. Burn shock of moderate severity in animals caused thermal burns of the second degree (21-23\%) by method (Regas \& Ehrlich, 1992).

Infusion of the investigated solutions in a dose of $10 \mathrm{ml} / \mathrm{kg}$ was carried out in aseptic conditions in the femoral vein for 5 minutes. One hour after the infliction of burn shock; the following infusions - once a day for 7 days. A catheter with a diameter of 1-2 mm, mounted in the femoral vein, was placed under the skin, and its lumen was filled with titrated heparin solution $(0.1 \mathrm{ml}$ of heparin per $10 \mathrm{ml}$ of $0.9 \% \mathrm{NaCl}$ solution) throughout the length after each injection of the solutions studied. Shaving of the animals, catheterization of major vessels, infliction of burns and decapitation of the animals were carried out under conditions of propofol anesthesia at a dose of $60 \mathrm{mg} / \mathrm{kg}$ intraperitoneally (Abdullahi et al., 2014).

Two schemes of pharmacological correction of opioid shock were investigated in this work: 1) $0.9 \%$ solution of $\mathrm{NaCl}$ (produced by the Kyiv JSC Biofarma) - control pathology; 2) the investigated drug lactoprotein with sorbitol - protein and salt complex containing as a colloidal basis donor albumin $-5.0 \%$, as well as polyatomic sorbitol $6.0 \%$, sodium lactate $-2.1 \%$, sodium chloride $-0.8 \%$, calcium chloride $0.01 \%$, potassium chloride $-0.0075 \%$, sodium bicarbonate $-0.01 \%$. Osmolarity of the drug - $1020 \mathrm{mOsmol} / \mathrm{l}$ (Registration certificate No 464/09-300200000 JSC Biofarma). Lactoprotein with sorbitol belongs to the group colloidal hyperosmolar solutions and is the closest in composition, transfusion potential and pharmacodynamic properties to blood plasma.

For electron microscopy, tracheotomy was performed in the rats under propofol anesthesia. After two-sided incisions along the intercostal spaces, the lungs collapsed, and then in the trachea about $1.5 \mathrm{ml}$ of $2.5 \%$ glutaraldehyde solution on the phosphate buffer was immediately administered. After opening of the chest in situ, the right lung was irrigated with a $2.5 \%$ glutaraldehyde solution for 15-20 minutes. Sections from the lower lobe of the right lung were cut into small blocks and continued to be fixed in the same solution for an hour. After standard wiring, the material was poured into an epoch. Ultra-thin sections of the lung tissue produced on ultramicrotome LKB-3 were prepared with uranyl acetate and lead citrate by the Reynolds method and the lung structure studied under the electron microscope PEM-125K (Regas \& Ehrlich, 1992).

\section{Results}

The longitudinal nuclei of endothelial cells and respiratory alveolocytes had invaginations, which are formed by the nuclear membranes. The wall of the aerogematous barrier was uneven due to edema and illumination of the cytoplasmic part of the respiratory epitheliocytes, their plasmolemma were wavy. Cell cytoplasm had a small number of pynocystic bubbles, there were separate vacuoles, and organelles were numb and destructively altered.

The thickness of the basement membrane was uneven, there were locally thickened areas. The cytoplasmic part of the endothelial cells had a different thickness, formed a leak in the lumen of the blood capillary. In the cytoplasm of the endothelial cells there were destructively altered organelles - hypertrophied mitochondria had places that were luminous matrix, damaged crystals, granular endoplasmic mesh channels were fragmented, thickened, on the surface of their membranes, there were few ribosomes.

Ultrastructure of type II alveolots was noticeably changed - there were small nuclei with uneven contours of the nuclear membrane. In the karyoplasm, there were many heterochromatin, they form a cluster and are located more along the nuclear membrane. In the cytoplasm, small numbers of mitochondria were hypertrophied, had a homogeneous matrix, in which the crystals were poorly visible, the channels of the granular endoplasmic mesh were deformed, uneven, on the surface of their membranes there were few ribosomes, and the cisternae in the dictyosomes of the Golgi complex were single. On the apical part of the plasmalema there were small microvilli, most of which is exposed. Such a state of secretory alveolocytes indicates their low functional activity (Fig. 1b). In the cytoplasm of the alveolar macrophages, many primary, small lysosomes and large secondary phagosomes were noted. The nucleus appeared diminished, the nuclear membranes formed invaginations, areas of heterochromatin were present in the karyoplasm. Cytoplasmic germs were present on separate areas of the surface, plasmolemma in them fuzzy, rippled, indicating a violation of the functional activity of alveolar macrophages (Fig. 1c). 

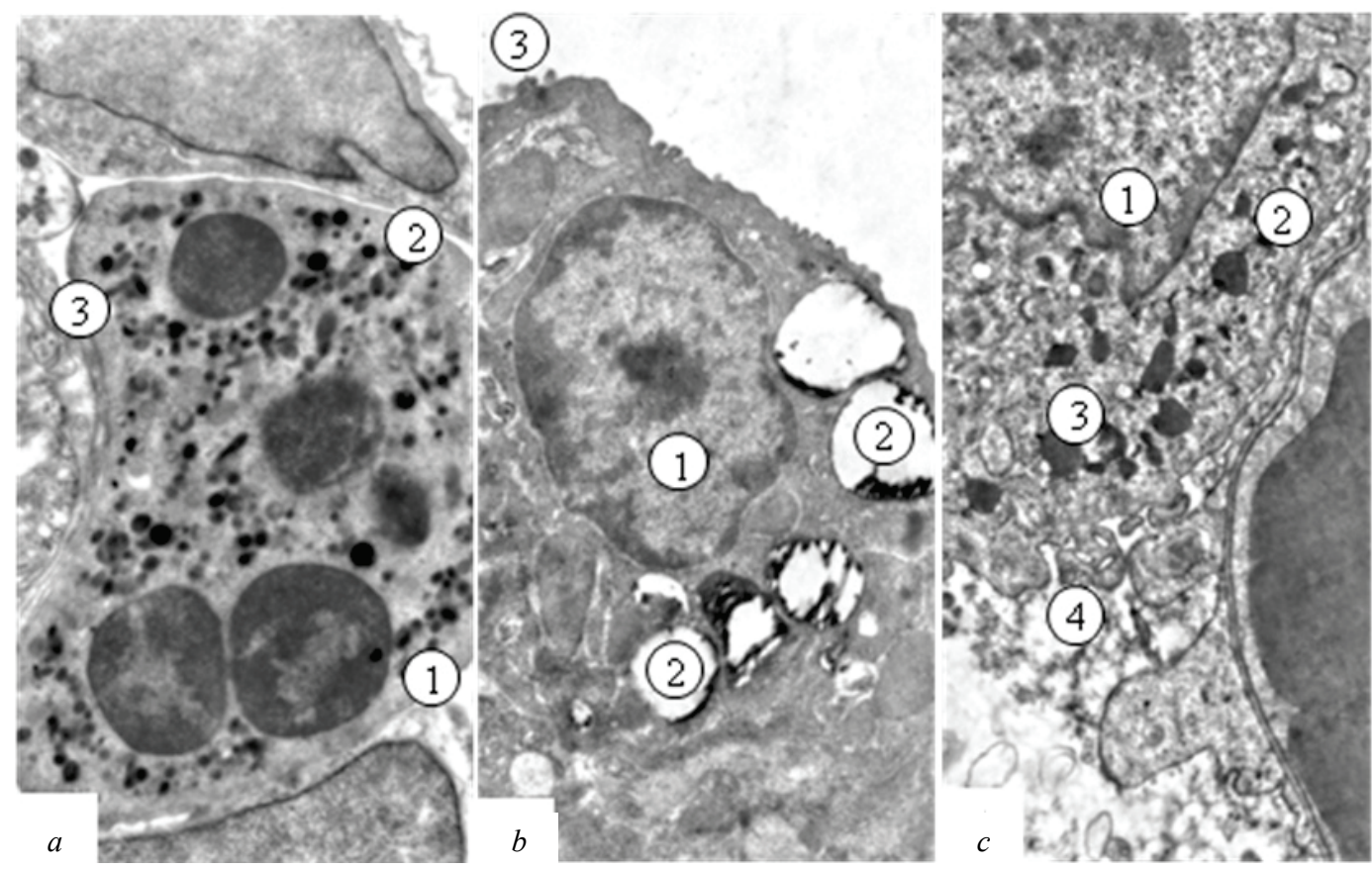

Fig. 1. Sub-microscopic changes in the respiratory lung unit for day 3 of burn shock when $0.9 \% \mathrm{NaCl}$ solution was administered (the scale bar was $100 \mu \mathrm{m}$ ): $a$-alveol: 1 - lumen of hemocapillary with blood cells, 2 - endothelial cell, 3 -aerogemic barrier; $b$-alveolocytes:

1 - nucleus, 2 - vacuole-shaped lamellar bodies, 3 - single microvilli on the surface of the cell; $c$-alveolar macrophage:

1 - kernel with invaginations of the cariolomas, 2 - primary lysosomes, 3 - secondary lysosomes, 4 - cytoplasmic growths

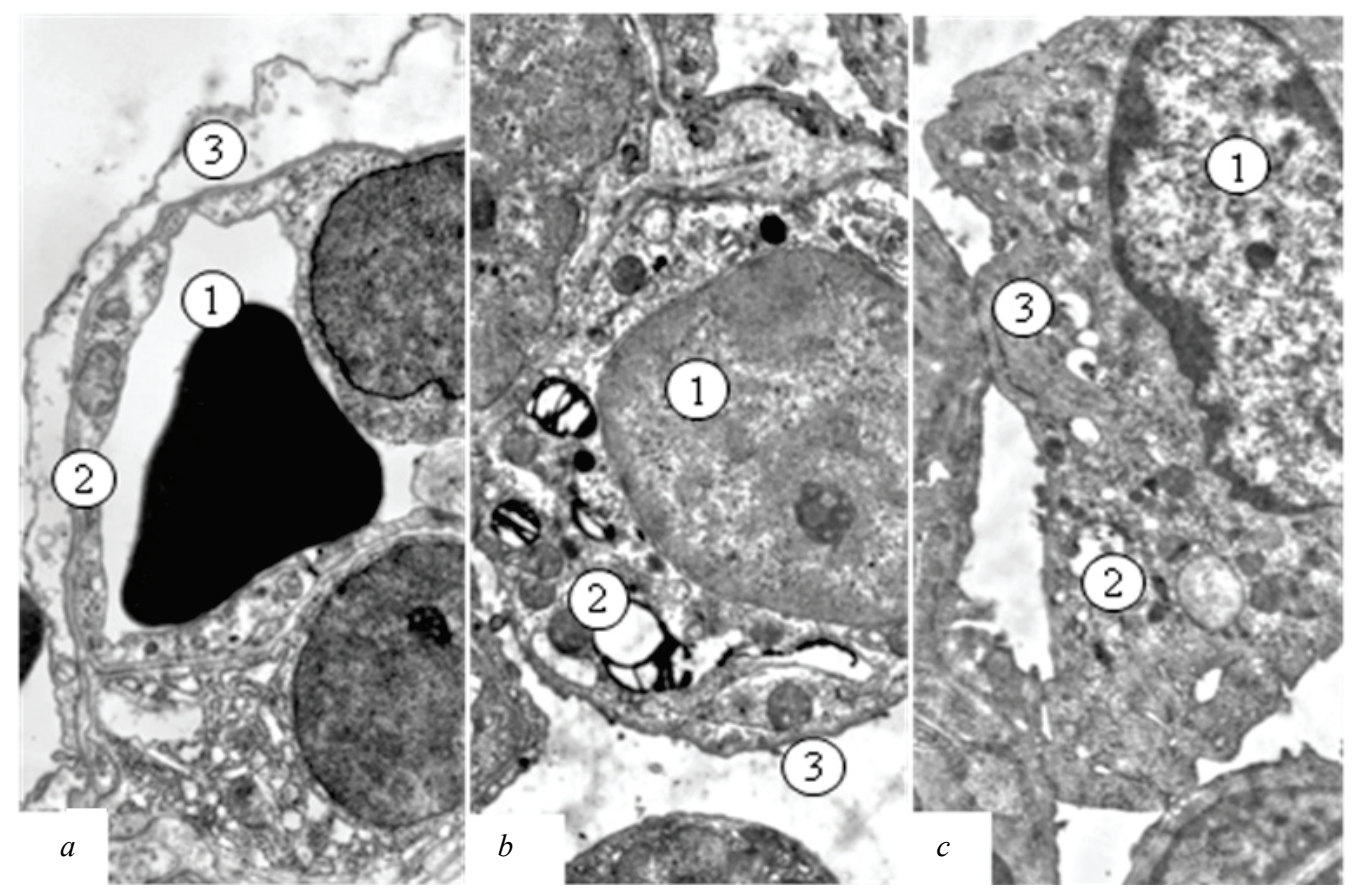

Fig. 2. Sub-microscopic changes in the respiratory lung unit on day 3 of burn shock when $0.9 \% \mathrm{NaCl}$ solution was injected (the scale bar was $100 \mu \mathrm{m}$ ): $a$-alveoli: 1 - lumen of haemocapillary with blood cells, 2 - endothelial cell, 3 -aerogemic barrier; $b$-alveolocytes:

1 - nucleus, 2 - vacuole-shaped lamellar bodies, 3 - single microvilli on the surface of the cell; $c$-alveolar macrophage:

1 - kernel with invaginations of the karyolemmas, 2 - primary lysosomes, 3 - secondary lysosomes, 4 - cytoplasmic growths

At day 7 of burn shock, with $0.9 \% \mathrm{NaCl}$ solution, significant changes were observed in the respiratory unit: part of the alveoli had considerably expanded blood flow of capillaries, which had platelets, neutrophils and altered erythrocytes. The wall of these alveoli had substantially thickened areas due to the of the edema cytoplasm of respiratory alveols and endothelial cells. In the aerohematic barrier there was a narrow basement membrane. The cytoplasmic parts of the endothelial cells were of different thicknesses. The nuclei of the endothelial cells were small, moderately osmeophilic. Organelles were single and damaged and occupied a small area. Respiratory epithelial cells had unevenly thickened, electron-trans- mitted cytoplasmic areas. Their outer membrane formed protuberance and invagination, pynocystic bubbles and caveolae were insufficient. Such a state of the aerohematic barrier, destructive changes in the structure's components indicates a significant deterioration of gas exchange in the respiratory lung (Fig. 2a). The ultrastructure of type II alveolocytes was significantly altered - there were violations of the karyolemmas, perinuclear space was expanding due to the detachment of the outer nuclear membrane, and a few nuclear pores in the cariolum. In the cytoplasm, the destruction of organelles was found: platelets were few, some of them were large with electron-translucent portions, contained little osmiumophi- 
lic material; in the mitochondria there was an osmiophilic homogenized matrix in which the crystals were not detected; unevenly expanded and partially fragmented tubules of a granular endoplasmic net and Golgi complexes resembling vacuoles; at the apical portion of plasmolem, single microvilli (Fig. 2b). In the cytoplasm of alveolar macrophages, there were many different electronic densities of phagos, primary lysosomes were small, they were small, rounded, osmotic, their plasmolem is uneven, but cytoplasmic germs were small (Fig. 2c), which indicates the accumulation of phagocyte material in them.

At day 3 of burn shock in the background of administration of a solution of lactobacilli with sorbitol, less damage was done to the structures of the respiratory lung than in the group of rats which was fed a $0.9 \%$ solution of $\mathrm{NaCl}$. The basement membrane looked like a light, moderate thickness of the tape, but in some areas it was not clearly contoured (Fig. 3a).

In uneven cytoplasmic areas of respiratory epitheliocytes, there were more pynocystic bubbles and caveoles, plasmolem formed bubbling and microvilli. Cytoplasmic areas of endothelial cells were of uneven thickness, they had few organelles (Fig. 3b).

During this period, the ultrastructure of type II alveolocytes and alveolar macrophages was similar to that of a group of animals that was fed a $0.9 \%$ solution of $\mathrm{NaCl}$ after infliction of the burn. Thus, in the cytoplasm of the secretory epithelial cells there were few platelets, they were large, electron-transparent, osmiophilic layered material was rarely observed. Mitochondria were small, osmiophilic, had a homogeneous matrix, crystals were poorly contoured.

On the apical part of the cells in plasmolomy there were microvilli, most of it was exposed. Such a state of secretory alveolocytes reflects their low functional activity. However, the nuclei of the secretory epitheliocytes had a rounded form, euchromatin predominated in their karyoplasm, and the kariolemmas were clearly contoured (Fig. 3b). This is a sign of the onset of intracellular regenerative processes. The cytoplasm of the alveolar macrophages had many primary small lysosomes and secondary phagos. Plasma cell cultures in selected areas were lacerated, fuzzy, cytoplasmic germs were present.

At day 7of burn shock in the lungs of rats which were injected with lactoprotein solution with sorbitol, the ultrastructure of the components of the aerohematic barrier had improved in comparison with 3 days. Luminosity of the hemocapillary parts was moderate, mainly with erythrocytes. Endothelial cells in their walls had elongated nuclei with invaginations of the nuclear membranes and clear contours. Their cytoplasmic regions were not wide-ranging, with moderate electron density.
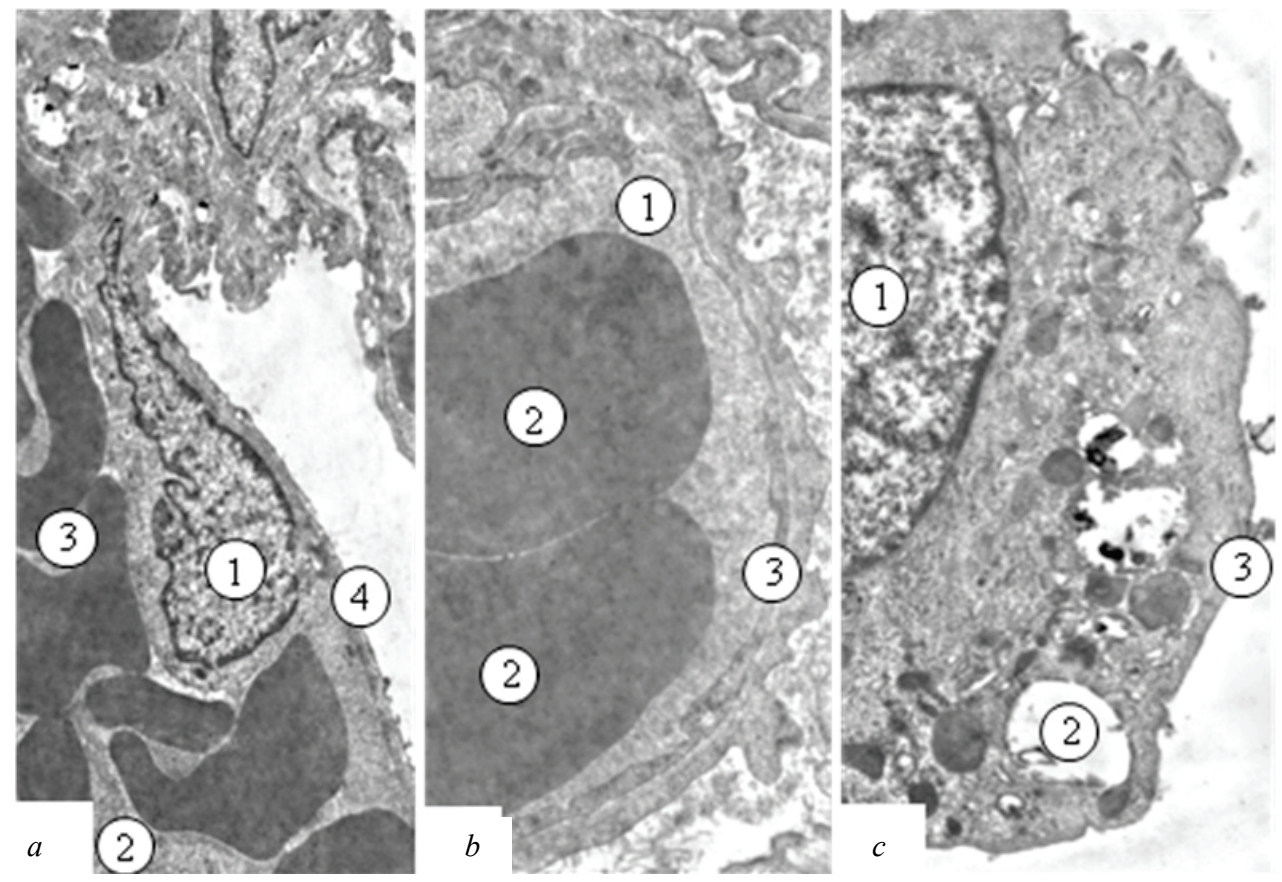

Fig. 3. Submicroscopic changes in the respiratory lung unit after 3 days of burn shock when lactoprotein and sorbitol were administered (the scale bar was $100 \mu \mathrm{m}$ ): $a$-alveoli: 1 -nucleus, 2 - lumen of hemocapillary, 3 - form elements of blood, 4 -aerohematic barrier; $b$-the walls of the alveoli: 1 - the lumen of the hemocapillary, 2 - red blood cells, 3 - aerohematic barrier; $c$-type II alveolocyte: 1 - nucleus, 2 - light secretory bodies, 3 - apical section of plasmolomy with microvilli

The basement membrane in the aerohematic barrier was relatively uniform, not wide, light, and from the side of the stroma was thickened and uneven. In the respiratory alveolocytes, cytoplasmic areas were of uneven thickness, plasmolemma, which limits wavelengths (Fig. 4a). However, the swelling of the cytoplasm and its illumination were not as significant as in this period when $0.9 \%$ solution of $\mathrm{NaCl}$ was introduced. In the part of cytoplasm segments of type I alveolocytes, many bubbles and caveolae were observed. Part of the blood capillaries remainned with enlarged, blood-filled lumens.

In type II alveolocytes, during this trial, a lower degree of damage to the nucleus and organelles in the cytoplasm was established and there were signs of a renewal of the secretory function of these cells. In the cytoplasm, we observed hypertrophied mitochondria with clear crystals, components of the Golgi complex, different size of secretory granules, which showed different densities, indicating their formation (Fig. 4b).

Near the wall of the alveoli, alveolar macrophages with many cytoplasmic germs were observed, some of them were branched. In the cytoplasm of the alveolar macrophages, there were many mitochondria, lysosomes present in small primary and larger secondary form. Such an ultrastructural organization of alveolar macrophages testifies to their functional activity and participation in protective reactions (Fig. $4 c$ ).

\section{Discussion}

Lungs play an important role in causing systemic homeostasis disorders against a background of burn shock (Shaver \& Bastarache, 2014; Nielson et al., 2017); increased permeability of the capillaries, their dilatation, a decrease in the volume of circulating blood, deterioration of rheological properties and the development of microthrombosis, decreased intrapulmonary blood flow as a result of hypoxic lung injury. However, further elucidation of the ultrastructural mechanism of homeostasis in the lungs will allow us to assess both early changes in the body and the effectiveness of the applied therapy. In general, the ultrastructural changes detected in the application of the physiological solution on days 3 and 7 indicate an inadequate effect of this type of infusion therapy in preventing cellular damage in the lungs, this is compared 
with the data regarding the development of pulmonary complications in the application of $0.9 \% \mathrm{NaCl}$ solution to burn patients and in experimental studies (Jacob et al., 2015; Sousse et al., 2015). The obtained data correspond to ultrastructural changes recorded in other experimental studies regarding cellular lung injury on the background of thermal burns (Zhang et al., 2018).

During electron microscopic examination of the respiratory part of the lungs on day 3 of burn shock against the background of the use of $0.9 \% \mathrm{NaCl}$ solution, reactive changes in the alveoli were determined, mainly destructive - necrotic, indicating a significant defeat of the organ in this period of thermal burn of the skin. We also revealed ultrastructural changes in alveolocytes which indicate their low functional activity. The changes observed correspond to the data obtained in other studies in the period of burn shock (Sousse et al., 2015; Zhang et al., 2018). At day 7 of burn shock, using $0.9 \%$ of $\mathrm{NaCl}$ solution, signs of progression of destructive changes in the components of the aerohematic barrier were observed, indicating the weak protective effect of $0.9 \%$ $\mathrm{NaCl}$ solution.

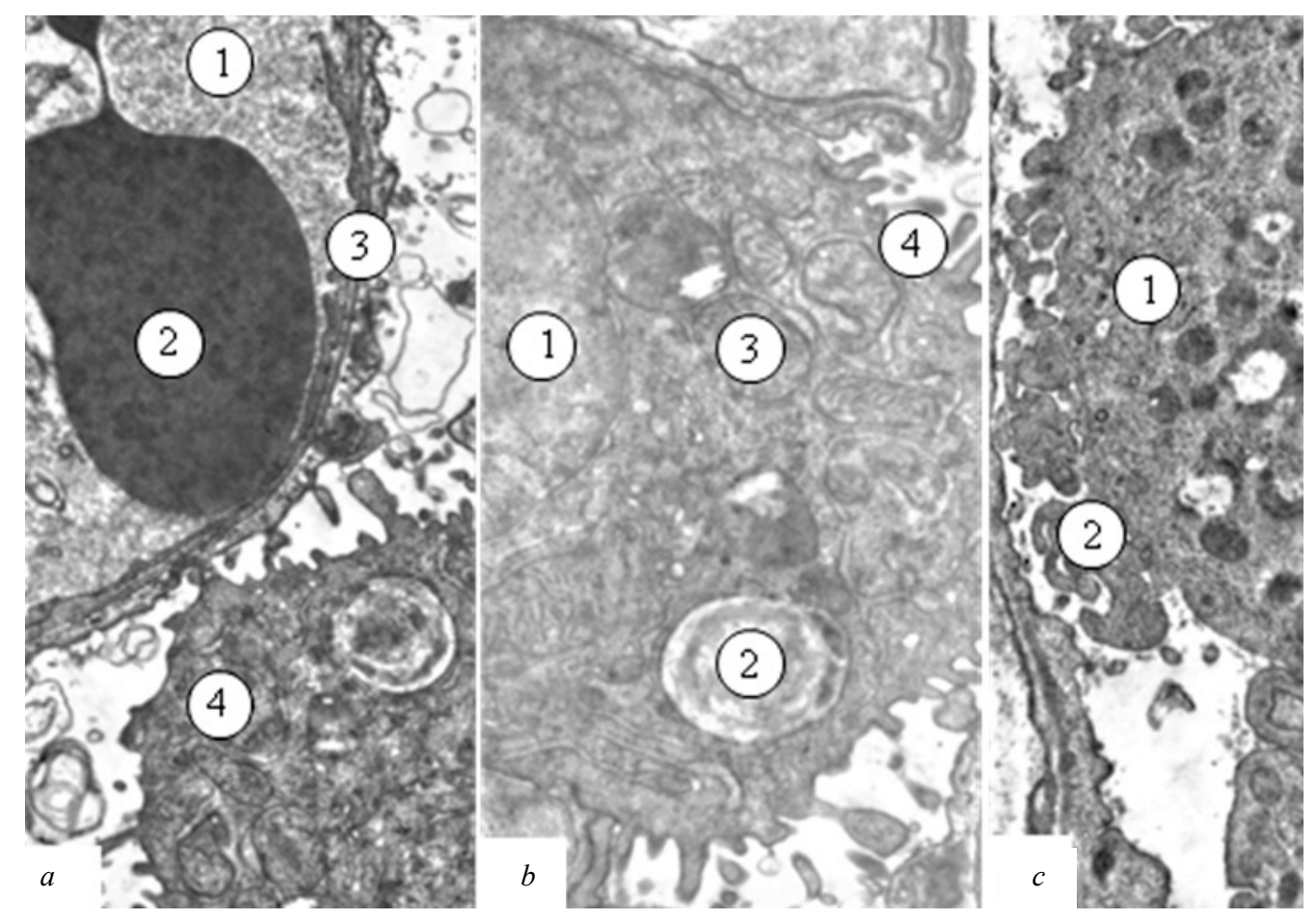

Fig. 4. Submicroscopic changes in respiratory lung part after 7 days of burn shock upon administration of lactobacilli with sorbitol: $a$-walls of the alveoli: 1 - lumen of the hemocapillary, 2 - erythrocyte, 3 - the wall of the alveoli, 4 - macrophage $\left({ }^{x} 9,000\right) ; b$ - type II alveolocyte:

1 - rounded nucleus, 2 - secretory granules, 3 -hypertrophied mitochondria, 4 - apical section of plasmolymy with microvilli ( $\left.{ }^{x} 15,000\right)$; $c$-alveolar macrophage: 1 - fragment of the cytoplasm, 2 - cytoplasmic germs $\left({ }^{x} 9,000\right)$

When studying the corrective effect of lactoprotein with sorbitol solution after 3 days of burn shock in rats, ultrastructural data did not differ from the group of rats when $0.9 \%$ solution of $\mathrm{NaCl}$ was introduced. However, the nuclei of the secretory epitheliocytes had a rounded form, euchromatin predominated in their karyoplasma, clearly defined nuclear membranes, well-expressed nucleoli are signs of the onset of intracellular regenerative processes. At day 7 under the action of the solution of lactoprotein with sorbitol, changes in the aerohematic barrier were less pronounced than against the background of $0.9 \% \mathrm{NaCl}$ solution. In type II alveolocytes there were signs of a renewal of the secretory function, the ultrastructural organization of alveolar macrophages testifyed to their functional activity and participation in protective reactions.

Thus, it was found at the subcellular level that the colloidal hyperosmolar solution of lactoproteins with sorbitol in the magnitude of the cytoprotective effect on lung cells with modelled burn shock prevails over the action of $0.9 \% \mathrm{NaCl}$ solution, which is consistent with the literature on the effect of this drug on other organs (Cherkasov et al., 2015). However, it should be noted that in contrast to other organs, where changes in organs are recorded from practically the first day of burn shock (Guminskiy et al., 2017). In our study, the greatest positive effect on the cellular structures of lungs of lactoprotein solution with sorbitol was established 7 days after skin burn. We can explain this by the fact that the lungs against the background of burn shock are an effector organ in which an inflammatory reaction with marked cellular damage develops (Chong et al., 2014), against which apoptosis is activated in damaged lung cells (Dong et al., 2015; Rose \& Chan, 2016). Therefore, the use of infusion solutions on lung cells is likely to exert its influence by activating synthetic processes that upgrade the cell popula- tion by replacing apoptotic cells as is established in other organs (Guo et al., 2015). It should also be noted that ultrastructural changes in the lungs with burn shock are noticed before the manifestation of clinical symptoms (Shaver \& Bastarache, 2014), which suggests it is precisely the effect of lactoprotein solution with sorbitol on lung cells, which in the long run may improve the results of the therapy of this pathological condition.

Such results of an experimental study allow us to differentiate the changes caused by burn shock from changes caused by the drug itself, proving the safety of the drug and the possibility of its use in this pathology. It is known (Perel et al., 2013) that prolonged use of only one crystalloid solution has a number of undesirable reactions: the need to increase the volume of infusion, insignificant duration of circulatory effect, cell dehydration, significant overload of the body with sodium ions, increased edema in the burn area, deepening systemic acidosis, decreased blood lactate levels, a sharp drop in potassium ions, and a negative inotropic effect on the myocardium. Therefore, the feasibility of their use is questioned because they are not able to align the hemodynamic status.

Unlike hypertonic solutions of sodium chloride, combined hyperosmolar solutions have a number of benefits that provide a comprehensive pharmacological action: detoxification, intestinal, energy, moderate dehydration, and are characterized by a lower risk of side effects: pulmonary edema, hypocoagulation, impaired filtration ability of the kidneys (Abdullahi \& Jeschke, 2014). Their effect is sufficiently studied (Perel et al., 2013), it is found that these solutions, which simultaneously affect the various parts of the burn shock, have a number of advantages over monotherapy: they can be used from the first hours of burn shock, 
minimizing the negative effects inherent in the early application of colloids, they include solutions for the correction of metabolic acidosis, which is an important factor in the overall damage to thermal skin burns (Shaver \& Bastarache, 2014; Nielson et al., 2017).

According to the results of an ultrastructural study, it was found in rats without burns which were injected a $0.9 \%$ solution of $\mathrm{NaCl}$ for 7 days and a lacto-protein with sorbitol that the structure of the aerohematic barrier remained unchanged: most of the lung cuts occupy the alveoli that formed alveolar strokes and alveolar sacs. In the cytoplasm of type II alveolocytes, a well-developed Golgi complex, elements of a granular endoplasmic net, numerous mitochondria, free ribosomes and polysomes, as well as lamellar bodies with surfactant, alveolar macrophages were introduced in the intervertebral septa, in their cytoplasm developed cisterns and electron-transparent vesicles of the Golgi complex, numerous mitochondria, primary and secondary lysosomes and vacuoles.

In contrast, the use of lactoprotein with sorbitol positively influenced the processes of renewal of cells in the lungs against the background of burn shock and intracellular structures more clearly from 7 days, which proves the advantage of this type of solution in the treatment of burn injury for tread effect on the lungs, which can and should be recommended for use according to modern guidelines (Snell et al., 2013; Abdullahi \& Jeschke, 2014; Kaddoura et al., 2017) requiring the use of solutions with rapid recovery of circulating blood volume and positive effect on microcirculation and tissue oxygenation. The use of such solutions optimizes the functioning of organs. The data we obtainned allow us to confirm the positive effect of the solution of lactobacillus with sorbitol on the ultrastructure of the lungs with thermal damage to the skin and the development of burn shock.

\section{Conclusions}

As a result of the study of the influence of the solution of lactoprotein with sorbitol on the ultrastructure of the tissues of the lungs in rats with burn shock, we can state the advantage of this preparation over the use of physiological $(0.9 \% \mathrm{NaCl})$ solution. In conditions of burn shock, the use of a solution of lactoprotein with sorbitol for 7 days is accompanied by a maximum pulmonoprotective action (as evidenced by ultrastructural changes in the lungs) in comparison with the control group using $0.9 \% \mathrm{NaCl}$ solution.

The positive effect of the solution of lactoprotein with sorbitol on the ultrastructure of the lungs in the event of burn injuries of the skin is realized through the restoration of the endothelium of blood vessels, hemocapillaries, basement membrane, fluid retention in the microcirculatory channel, improvement of the aerohematic barrier, stimulation of the activity of alveolar macrophages and the secretory function of type II alveolocytes. The most distinct changes were observed at 7 days after induction of burn shock in the lungs of the rats which were injected with a solution of lactoprotein with sorbitol, the ultrastructure of the components of the aerohematic barrier improved compared with the first 3 days of the experiment using a similar solution.

The study of functional, biochemical and molecular genetic parameters that characterize the state of the aerohematic barrier following the use of a solution of lactoprotein with sorbitol in patients with a burn injury to the skin will allow a comprehensive assessment of the mechanisms of the pulmonary protective effect of this drug and experimenttally substantiate the feasibility of its use in clinical practice for pharmacocorrection of burn shock.

\section{References}

Abdullahi, A., \& Jeschke, M. G. (2014). Nutrition and anabolic pharmacotherapies in the care of burn patients. Nutrition in Clinical Practice, 29(5), 621-630.

Abdullahi, A., Amini-Nik, S., \& Jeschke, M. (2014). Animal models in burn research. Cellular and Molecular Life Sciences, 71(17), 3241-3255.
Cherkasov, V. G., Kovalchuk, A. I., Dzevulskaya, I. V., \& Cherkasov, E. V. (2015). Evaluation of the effect of infusion of composite hyperosmolar solutions on the structure of the neuroimmunoendocrine system organs in burn disease. European International Journal of Science and Technology, 4(9), 51-61.

Chong, S. J., Wong, Y. C., Wu, J., Tan, M. H., Lu, J., \& Moochhala, S. M. (2014). Parecoxib reduces systemic inflammation and acute lung injury in burned animals with delayed fluid resuscitation. International Journal of Inflammation, 2014, 972645 .

Cox, R. A., Jacob, S., Andersen, C. R., Mlcak, R., Sousse, L., Zhu, Y., \& Hawkins, H. K. (2015). Integrity of airway epithelium in pediatric burn autopsies: Association with age and extent of burn injury. Burns, 41(7), 1435-1441.

Directive 2010/63/EC of the European Parliament and of the Council of 22 September 2010 on the protection of animals used for scientific purposes (2010). Official Journal of the European Union, 276, 33-79.

Dong, Z. W., Chen, J., Ruan, Y. C., Zhou, T., Chen, Y., Chen, Y., \& Peng, Y. Z. (2015). CFTR-regulated MAPK/NF- $\mathrm{B}$ signaling in pulmonary inflammation in thermal inhalation injury. Scientific Reports, 5, 15946.

Fuzaylov, G., Anderson, R., Knittel, J., \& Driscoll, D. N. (2015) Global health: Burn outreach program. Journal of Burn Care and Research, 36(2), 306-309.

Gamelli, L., Mykychack, I., Kushnir, A., Driscoll, D. N., \& Fuzaylov, G. (2015) Targeting burn prevention in Ukraine: Evaluation of base knowledge in burn prevention and first aid treatment. Journal of Burn Care and Research, 36(1), 225-231.

Glik, J., Kawecki, M., Kitala, D., Klama-Baryła, A., Łabuś, W., Grabowski, M., \& Kasperczyk, A. (2017). A new option for definitive burn wound closurepair matching type of retrospective case-control study of hand burns in the hospitalised patients group in the Dr Stanislaw Sakiel Centre for Burn Treatment between 2009 and 2015. International Wound Journal, 14(5), 849-855.

Guminskiy, Y. I., Gunas, I. V., Ocheretna, N. P., \& Bashinska, O. I. (2017). Micromorphometric changes in rats' spleen in the first 7 days after skin burns and under application of infusion solutions. Reports of Morphology, 23(2), 240-244.

Guo, S.-X., Fang, Q., You, C.-G., Jin, Y.-Y., Wang, X.-G., Hu, X.-L., \& Han, C.-M. (2015). Effects of hydrogen-rich saline on early acute kidney injury in severely burned rats by suppressing oxidative stress induced apoptosis and inflammation. Journal of Translational Medicine, 13, 183.

Herold, S., Gabrielli, N. M., \& Vadász, I. (2013). Novel concepts of acute lung injury and alveolar-capillary barrier dysfunction. American Journal of Physiology - Lung Cellular and Molecular Physiology, 305(10), L665-L681.

Jacob, S., Zhu, Y., Kraft, R., Cotto, C., Carmical, J. R., Wood, T. G., \& Cox, R. A. (2015). Physiologic and molecular changes in the tracheal epithelium of rats following burn injury. International Journal of Burns and Trauma, 5(1), 36-45.

Kaddoura, I., Abu-Sittah, G., Ibrahim, A., Karamanoukian, R., \& Papazian, N. (2017). Burn injury: Review of pathophysiology and therapeutic modalities in major burns. Annals of Burns and Fire Disasters, 30(2), 95-102.

Nielson, C. B., Duethman, N. C., Howard, J. M., Moncure, M., \& Wood, J. G. (2017). Burns: Pathophysiology of systemic complications and current management. Journal of Burn Care and Research, 38(1), e469-e481.

Perel, P., Roberts, I., \& Ker, K. (2013). Colloids versus crystalloids for fluid resuscitation in critically ill patients. Cochrane Database of Systematic Reviews, 2(2), CD000567.

Porter, C., Tompkins, R. G., Finnerty, C. C., Sidossis, L. S., Suman, O. E., \& Herndon, D. N. (2016). The metabolic stress response to burn trauma: Current understanding and therapies. Lancet, 388(10052), 1417-1426.

Regas, F. C., \& Ehrlich, H. P. (1992). Elucidating the vascular response to burns with a new rat model. The Journal of Trauma, 32(5), 557-563.

Rose, L. F., \& Chan, R. K. (2016). The burn wound microenvironment. Advances in Wound Care, 5(3), 106-118.

Rowan, M. P., Cancio, L. C., Elster, E. A., Burmeister, D. M., Rose, L. F., Natesan, S., \& Chung, K. K. (2015). Burn wound healing and treatment: Review and advancements. Critical Care, 19, 243.

Shaver, C. M., \& Bastarache, J. A. (2014). Clinical and biological heterogeneity in acute respiratory distress syndrome: Direct versus indirect lung injury. Clinics in Chest Medicine, 35(4), 639-653.

Snell, J. A., Loh, N.-H. W., Mahambrey, T., \& Shokrollahi, K. (2013). Clinical review: The critical care management of the bum patient. Critical Care, 17(5), 241.

Sousse, L. E., Herndon, D. N., Andersen, C. R., Zovath, A., Finnerty, C. C., Mlcak, R. P., Cox, R. A., Traber, D. L., \& Hawkins, H. K. (2015). Pulmonary histopathologic abnormalities and predictor variables in autopsies of burned pediatric patients. Burns, 41(3), 519-527.

Zhang, D., Chang, Y., Han, S., Yang, L., Hu, Q., Yu, Y., \& Chai, J. (2018). The microRNA expression profile in rat lung tissue early after burn injury. Turkish Journal of Trauma and Emergency Surgery, 24(3), 191-198. 